\title{
Polystyrene grafted carbon black synthesis via in situ solution radical polymerization in ionic liquid
}

\author{
Xueli Wu • Jianhui Qiu • Peng Liu • Eiichi Sakai • Lin Lei
}

Received: 4 January 2013 / Accepted: 6 May 2013 /Published online: 17 May 2013

(C) The Author(s) 2013. This article is published with open access at Springerlink.com

\begin{abstract}
DENKA BLACK/polystyrene nanocomposites (DB/PS) were successfully prepared through a facile strategy. Some of the styrene molecules were grafted and polymerized onto the surface of the DB via the in situ free radical addition process in ionic liquid, and others selfpolymerized and coated on the polystyrene grafted DB. The ionic liquid improves the grafting percentage because of its high viscosity. The electrical properties of polymer composites before and after the grafting were measured. It was found that the electrical conductivity of the DB/PS composites decreased with increasing amounts of monomer styrene and initiator 2, 2'-azobis (isobutyronitrile) (AIBN). The microstructure, morphology and thermal properties of the composites were characterized by Fourier transforminfrared spectroscopy (FT-IR), thermogravimetric analysis (TGA), scanning electron microscopy (SEM), transmission electron microscopy (TEM) and Raman spectroscopy. The results proved that the polystyrene had been successfully grafted onto the DB surface.
\end{abstract}

Keywords Nanocomposites · Electrical properties · Thermal properties $\cdot$ Microstructure $\cdot$ Ionic liquid

\section{Introduction}

Carbon black (CB) was first manufactured in the US from natural gas on soap-stone plates in 1872 [1], and it was widely

X. Wu J. Qiu $(\bowtie) \cdot$ E. Sakai $\cdot$ L. Lei

Department of Machine Intelligence and Systems Engineering, Faculty of Systems Engineering, Akita Prefectural University, Akita 015-0055, Japan

e-mail: qiu@akita-pu.ac.jp

P. Liu

Institute of Polymer Science and Engineering, College of Chemistry and Chemical Engineering, Lanzhou University, Gansu 730000, China used since when mixed with rubber, the mechanical properties of rubber were significantly improved [2]. CB refers to a group of industrial products including acetylene black, furnace black, channel black, thermal black and lampblacks. They are composed essentially of elemental carbon in the form of near-spherical particles of colloidal sizes, combined mainly into particle aggregates obtained by partial ignition or thermal decomposition of hydrocarbons [2]. CB is considered to have high prospects for use as filler materials for future polymer composites due to its high aspect ratio and superior electrical and thermal conductivities. Also, CB can be spread in a layer of nanometer scale thickness. Recently, there has been increased interest in composites of $\mathrm{CB}$ and polymers [3-5]. However, processing of CB has been limited by its poor solubility or dispersibility in most common solvents due to the strong Van der Waals attraction [6].

Many methods have been used to modify the CB properties, such as physical coating, chemical covalent bonding and non-covalent bonding. Among these methods, functionalization of $\mathrm{CB}$ with polymers is gaining wide interest, because the polymer chains not only improve the solubility of the $\mathrm{CB}$ but also enhance the thermodynamic stability, electrical conductivity and mechanical properties [7-10]. Moreover, this functionalization can lead to formation of $\mathrm{CB} /$ Polymer composites which are useful materials. Celzard et al. [11] were the first to investigate the conductive behavior of $100 \mu \mathrm{m}$ thick composite films composed of epoxy resin and expanded graphite flakes with the aspect ratio of about $100 \mathrm{~nm}$ and the filler volume fraction of around $1 \%$. Bouazzaoui's group reported microwave measurements on a large series of epoxy samples based on the CB-filled diglycidylic ether of bisphenol A (DGEBA) [12, 13]. They concluded that fitting the experimental data with mixing law and effective medium equation predictions had limited applicability because the mean field model of the predictions assumed a given microstructure for the composite material. Another important property of CB is its good 
anti-friction characteristic. Many researches study on the friction and wear behavior have been reported focused on the CB-reinforced metals and plastics [14-17].

Moreover, ionic liquids (ILs) have applications in electrochemical, synthetic, catalytic, and separations technologies because they are high in viscosity, have a large temperature range in which they can be used, and they are environmentally friendly [18]. ILs are composed solely of ionized species and have been proven to be useful solvents in polymer chemistry and engineering $[19,20]$.

In this work, DENKA BLACK (DB, a trade name for acetylene black) was chosen as a type of CB to prepare DB/PS nanocomposites via an in situ free radical addition process using IL as solvent because of its nonvolatile, nonflam-mable, and thermally stable characteristics [20]. Styrene was grafted and polymerized onto the DB surface. The polystyrene-grafted DB agglomerated and separated from the IL because the polystyrene cannot be suspended in the ionic liquid. The results of FT-IR, TGA, TEM and Raman spectrum measurements proved that the polystyrene was successfully grafted onto the DB surface. Electrical resistance measurements showed that the electrical conductivity of DB/PS nanocomposites was much higher than that of pure polystyrene $\left(10^{-16} \mathrm{~S} / \mathrm{cm}\right)$. The effects of initiator addition and the amount of styrene on the grafting of DB were evaluated by changing several experimental parameters.

\section{Experimental}

\section{Materials}

DENKA BLACK (DB) was obtained from Showa Denko Company, Tokyo, Japan. DB is acetylene black, a type of carbon black, obtained from thermal decomposition of acetylene. It is highly purified and extremely conductive. Styrene was purchased from NakaraIntesuku Company, Japan. 2, 2'azobis (isobutyronitrile) (AIBN) was purchased from Aladdin Reagent in Shanghai, China, and re-crystallized in ethanol. The IL (1-hexyl-3-methylimidazolium tetrafluroborate) was bought from Flute Cypress Chemistry Technology Company, Shanghai, China.

\section{Characterization}

Microstructure of grafted DB was determined by Fourier transform-infrared spectroscopy (FT-IR) (Model IRT-7000; JASCO, Japan). The measurements were carried out with the $\mathrm{KBr}$ pellet method, and were conducted with an aperture of $50 \mu \mathrm{m} \times 50 \mu \mathrm{m}$ and 30 scans in the transmission mode. The electrical resistance of nanocomposites was determined with a specific resistance meter (Model MCP-HT450, Mitsubishi Chemical Analytech). The pieces were pressed into slice with the thickness of about $0.5 \mathrm{~mm}$ with a hot press (Imoto Machinery Co., Ltd., Kyoto, Japan). The pressing conditions were: pressure, $200 \mathrm{kgf} / \mathrm{cm}^{-2}$, as shown on a pressure gage; temperature, $170{ }^{\circ} \mathrm{C}$; and time, $5 \mathrm{~min}$. The morphology was characterized by scanning electron microscopy (SEM, Model S-4300, Hitachi Ltd., Tokyo, Japan). SEM specimens were made by sputter coating the broken fracture surface of a pressed piece with gold to provide enhanced conductivity and then attaching the sputter coated piece onto the electrical double-tape on a metal plate. An automatic thermal analyzer (Shimadzu DTG60) was used for the thermogravimetric analysis (TGA). TGA conditions were: heating rate, $10{ }^{\circ} \mathrm{C} / \mathrm{min}$ from room temperature to $800{ }^{\circ} \mathrm{C}$; air atmosphere. The microstructure of the composites was determined by transmission electron microscopy (TEM) (Model H-8110, Hitachi Ltd.). To prepare TEM specimens, composite powders were dispersed in toluene in an ultrasonic bath for $30 \mathrm{~min}$, and then deposited on a copper grid covered with a perforated carbon film.

In situ solution polymerization

A certain amount of $\mathrm{DB}$ and $\mathrm{IL}(10 \mathrm{~mL})$ were mixed in a $100 \mathrm{~mL}$ round bottom flask and subjected to ultrasonic scattering for about $4 \mathrm{~h}$. Then a certain amount of styrene was added and the mixture was subjected to ultrasonic for another $2 \mathrm{~h}$. The flask was heated to a certain temperature in an oil bath and then a certain amount of initiator AIBN was added. The mixture was reacted for $12 \mathrm{~h}$ until the polystyrene grafted onto the surface of DB and separated from the IL. The IL solution was filtered to obtain the solid product. Then, the solid product was soaked in toluene for about a day and precipitated with ethanol, and air dried.

\section{Results and discussion}

In the present work, the polystyrene was grafted onto the DB surface via in situ solution polymerization in IL. In the polymerization process, the polymer chain radicals that reacted with the $\mathrm{C}=\mathrm{C}$ bonds of the $\mathrm{DB}$ were produced by three approaches: (1) the in situ radical polymerization of the monomers in the presence of the DB after initiation with initiator; (2) a controlled/'living' radical polymerization; and (3) the polymer chain transfer reaction [21]. Then the soxhlet extraction was carried out for some of the DB/PS nanocomposites to remove the polystyrene which was just coated on the polystyrene grafted DB.

Percentage grafting (PG) and electrical conductivity

After the polymerization of styrene onto the DB surface, the products were hot-pressed into slices for electrical conductivity 
Table 1 Percentage of grafting $(\mathrm{PG} \%)$ and surface resistance of $\mathrm{DB} / \mathrm{PS}$ at different conditions

*DB/PS11: with no ionic liquid

\begin{tabular}{lllllcl}
\hline No. & $\mathrm{DB}(\mathrm{g})$ & $\mathrm{St}(\mathrm{ml})$ & $\mathrm{AIBN}(\mathrm{g})$ & $\mathrm{T}\left({ }^{\circ} \mathrm{C}\right)$ & $\mathrm{PG}(5 \%)$ & $\rho(\mathrm{S} / \mathrm{cm})$ \\
\hline DB/PS1 & 0.2 & 10 & 0.0500 & 90 & 49.46 & $0.87 \times 10^{-10}$ \\
DB/PS2 & 0.2 & 10 & 0.0250 & 90 & 5.89 & $0.32 \times 10^{-8}$ \\
DB/PS3 & 0.2 & 10 & 0.0125 & 90 & 3.77 & $0.18 \times 10^{-10}$ \\
DB/PS4 & 0.2 & 10 & 0.0063 & 90 & 3.05 & $0.22 \times 10^{-11}$ \\
DB/PS5 & 0.2 & 10 & 0.1000 & 90 & 32.4 & $0.91 \times 10^{-11}$ \\
DB/PS6 & 0.2 & 5 & 0.0250 & 90 & 20.47 & $040 \times 10^{-5}$ \\
DB/PS7 & 0.2 & 2.5 & 0.0125 & 90 & 17.86 & $0.26 \times 10^{-4}$ \\
DB/PS8 & 0.2 & 1.25 & 0.0063 & 90 & 9.46 & $0.07 \times 10^{-3}$ \\
DB/PS9 & 0.2 & 10 & 0.0500 & 80 & 10.73 & $0.70 \times 10^{-10}$ \\
DB/PS10 & 0.2 & 10 & 0.0500 & 70 & 8.42 & $0.12 \times 10^{-6}$ \\
DB/PS11 & 0.2 & 10 & 0.0500 & 90 & 3.55 & $0.03 \times 10^{-11}$ \\
\hline
\end{tabular}

measurements. Due to the innate electrical conductivity of DB, the electrical conductivity of DB/PS nanocomposites was much higher than that of pure polystyrene $\left(10^{-16} \mathrm{~S} / \mathrm{cm}\right)$. The DB/PS nanocomposites showed semiconductor characteristics. Table 1 shows the $\mathrm{PG}$ and electrical conductivity of $\mathrm{DB} / \mathrm{PS}$ nanocomposites. The $\mathrm{PG}$ values were calculated from the TGA weight loss in the temperature range of $260-460{ }^{\circ} \mathrm{C}$. Both the $\mathrm{PG}$ and the conductivity first increased and then decreased with increasing amount of AIBN (DB/PS1-5). This phenomenon was also seen in Fig. 1. It occurred because with the increase of initiator, the polystyrene formed faster, so the probability of the addition reaction of the free radicals onto the DB increased, and the dispersibility improved accordingly. However, with too much initiator added, the coupling of the free radicals with each other to terminate the polymerization might become the dominant reaction [21], which led to the low PG. Furthermore, the PG was the maximum when the AIBN was $0.05 \mathrm{~g}$, but the maximum conductivity occurred when the AIBN was $0.025 \mathrm{~g}$. This might be due to more PS was grafted onto the DB surface, meanwhile more Sp2 have been changed to $\mathrm{Sp} 3$, which can destroy the structure of DB. In order to study

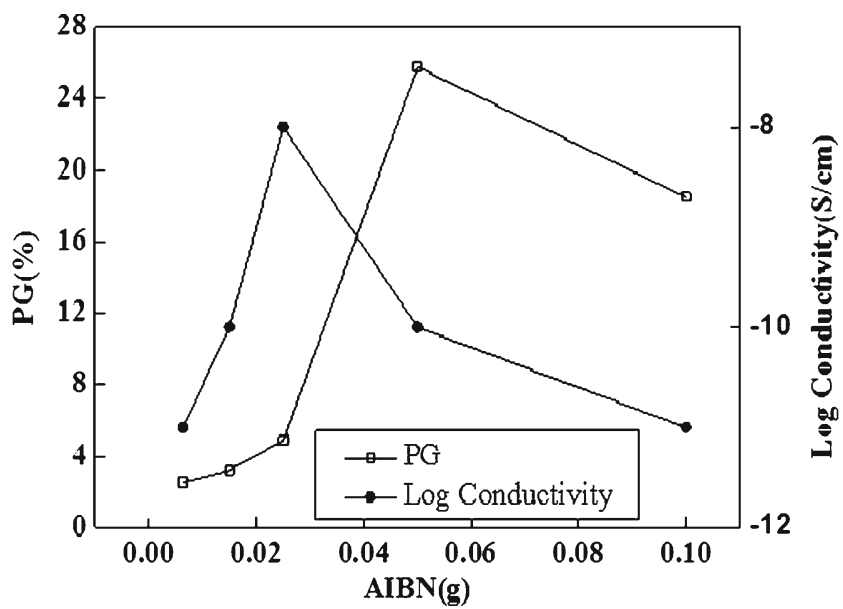

Fig. 1 AIBN influence on the PG and electrical conductivity of samples the effect of the amount of styrene on conductivity and $\mathrm{PG}$, four specimens (DB/PS1, DB/PS6, DB/PS7 and DB/PS8) were prepared with different amounts of Styrene, in which the ratio of AIBN and styrene fixed to $0.5 \%$ because the PG has a maximum at this ratio. It was obvious that the conductivity decreased and the PG increased with increasing amount of monomer as shown in Fig. 2, because the more monomer was added, more chances of polymer was grafted on the DB surface, and the greater the consumption of DB. The PG decreased and the conductivity increased with the decrease of temperature (specimens DB/PS1, DB/PS9 and DB/PS10) because the optimal temperature of AIBN initiator is around $90{ }^{\circ} \mathrm{C}$. It was also found that both the PG and the conductivity of nanocomposites which were prepared in IL were better than those values when the specimens were prepared with no IL added (DB/PS1 and DB/PS11). This was because the styrene was well dispersed in IL which contributed to the uniform mixing of DB and styrene.

Figure 3 shows the relationship between PG and conductivity of nanocomposites. The conductivity first increased and then decreased with increasing PG. This was due to the

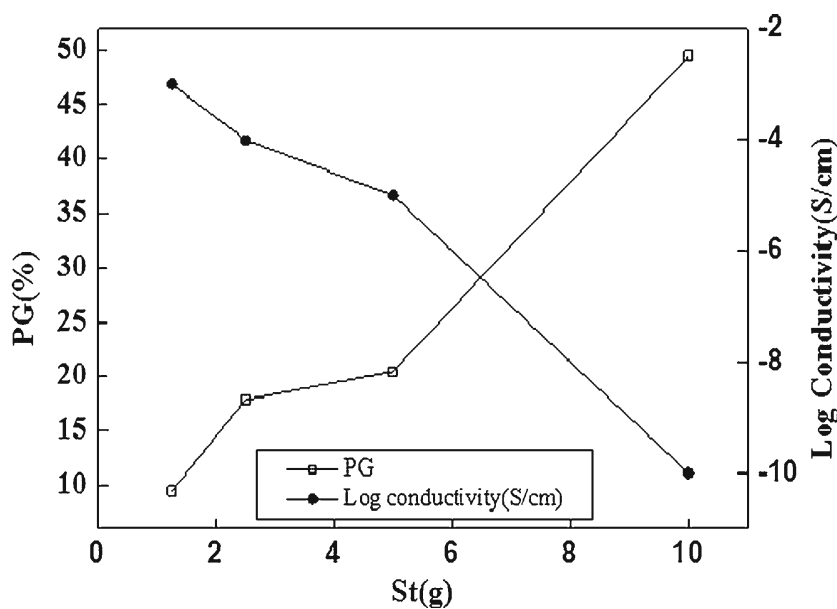

Fig. 2 Monomer influence on the PG and electrical conductivity of samples 


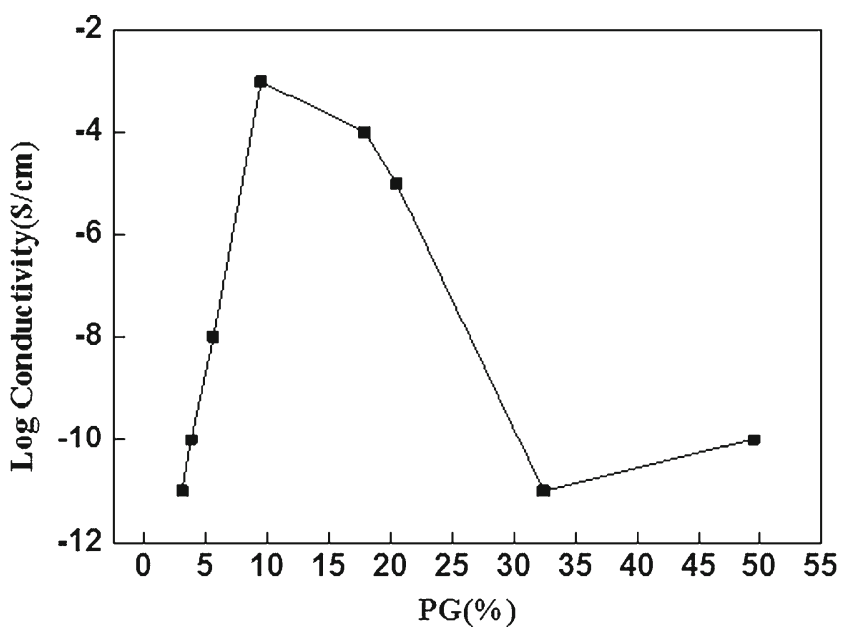

Fig. 3 The relationship between PG and electrical conductivity

poor dispersibility of DB in PS matrix when the PG was low, and the larger consumption of DB when the PG was high. The dispersibility can also be certified in SEM images as shown in Fig. 10.

\section{FI-IR spectroscopy measurements}

FT-IR spectroscopy measurements are important in the detection of the structure of DB/PS nanocomposites. Figure 4 shows the asymmetrical and symmetrical stretching vibrations of $-\mathrm{CH}_{2}$ at 2916 and $2854 \mathrm{~cm}^{-1}$, the flexural vibrations of $-\mathrm{CH}_{2}$ at $1446 \mathrm{~cm}^{-1}$, the characteristic C-C ring stretching vibration at $1600 \mathrm{~cm}^{-1}$ and the benzene ring plane stretching vibration of $-\mathrm{CH}$ at 756 and $706 \mathrm{~cm}^{-1}$ for the DB/PS1 specimen nanocomposites. All of these vibrations did not appear in the FT-IR spectrum of pure DB. This indicates that the polystyrene chains had been grafted onto the DB surface via the free radical addition polymerization.

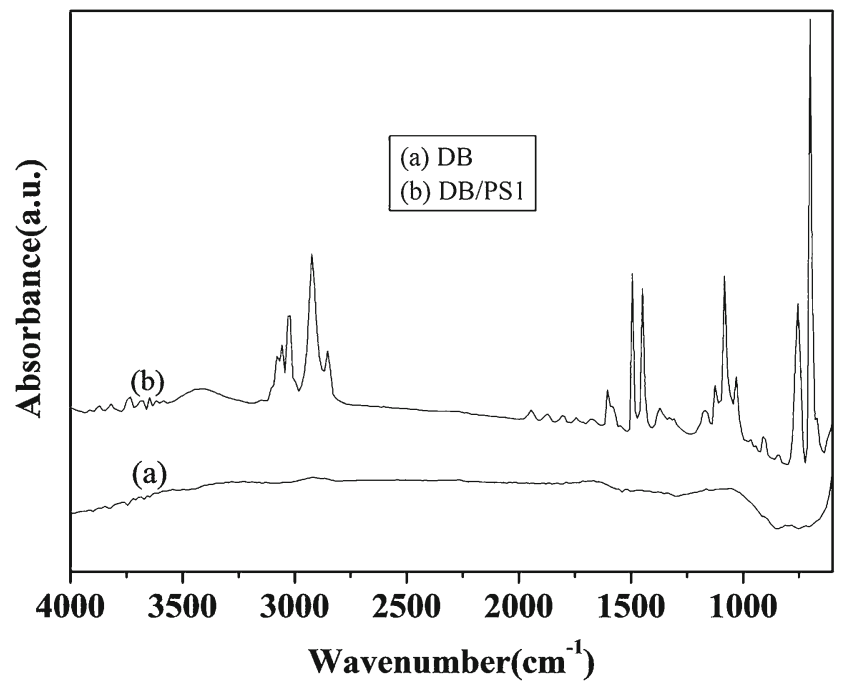

Fig. 4 FTIR spectra of (a) pure DB and (b) DB/PS1

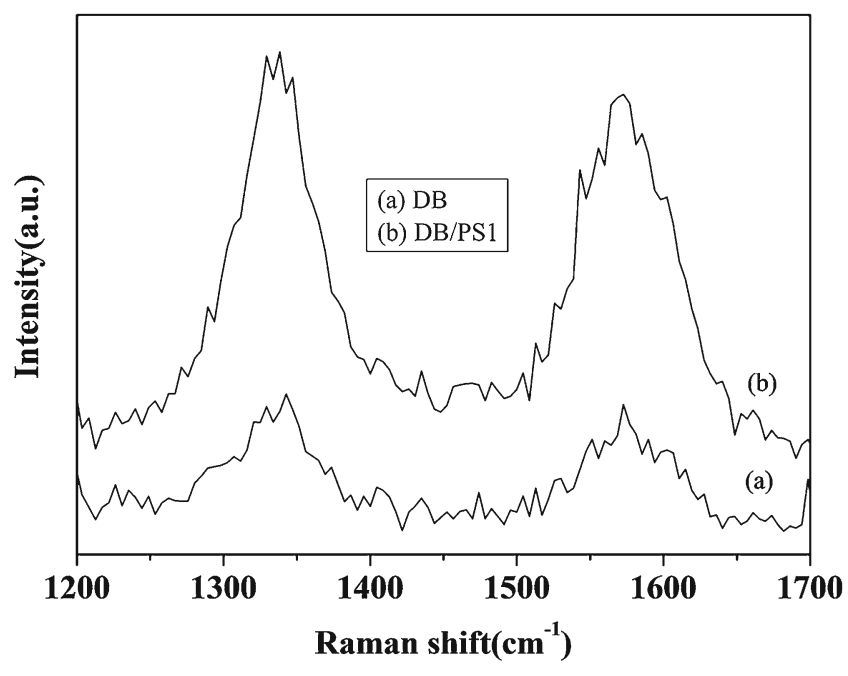

Fig. 5 Raman spectrum of (a) pure DB and (b) DB/PS1

Raman spectroscopy measurements

Historically, Raman spectroscopy has played an important role in characterizing the structure of carbon materials [22]. As shown in Fig. 5, the Raman spectrum of the pure DB shows a characteristic D band at $1336 \mathrm{~cm}^{-1}$ (defects/disorder-induced modes) and a $\mathrm{G}$ band at $1572 \mathrm{~cm}^{-1}$ (in-plane stretching tangential modes) with an intensity ratio (ID/IG) of 1.01. As for $\mathrm{DB} / \mathrm{PS} 1$, the ID/IG intensity ratio increased to 1.12 . The change in ID/IG intensity could also be observed from the change of the peak height. For the pure DB, the D band is about the same as the G band. However, for DB-PS1, the D band has a higher intensity than the $G$ band. The results showed that the in situ solution free radical polymerization had led to an increase in the number of defects in the DB nanoparticles.

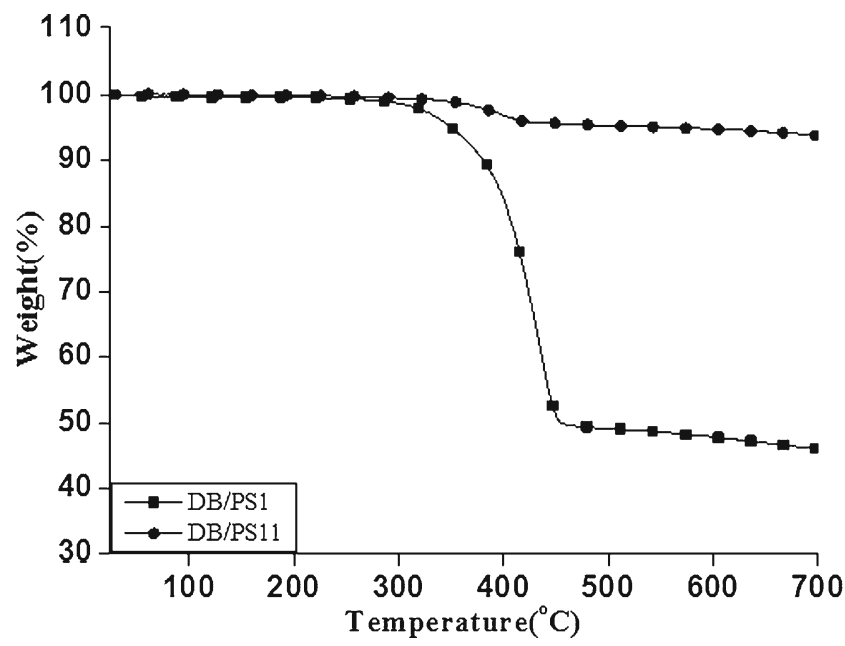

Fig. 6 The effect of ion liquid on the grafting percentage of nanocomposites 


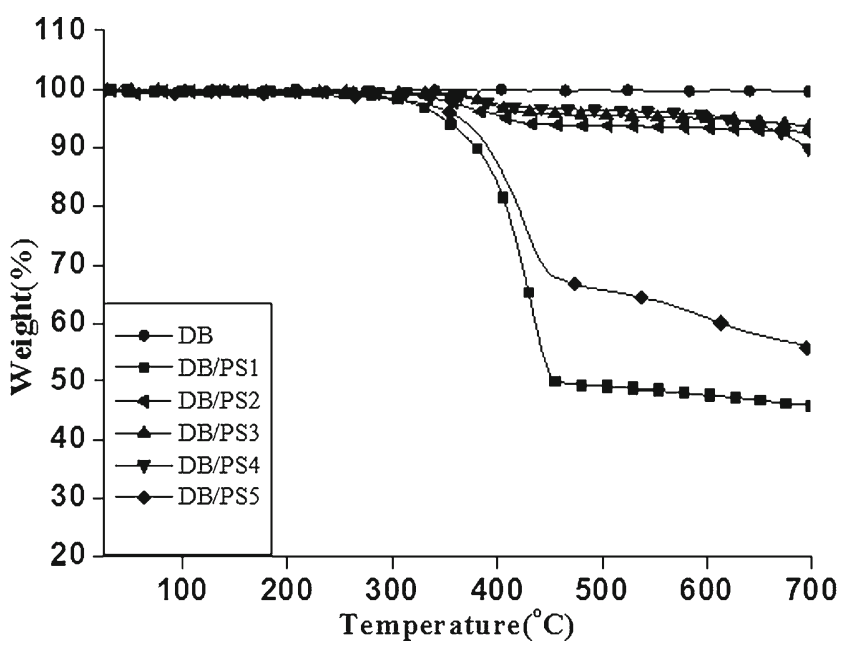

Fig. 7 The TGA curves of DB/PS nanocomposites with different amounts of monomer

\section{Advantage of IL use}

In order to prove that the IL can raise the grafting percentage of PS on the DB surface, DB/PS11 specimen was prepared without IL and other conditions the same as for DB/PS1. As shown in Fig. 6, the PG of DB/PS1 was much more than that of DB/PS11. This might be due to the fact that the lifetime of the surface radicals formed was prolonged because of the high viscosity of IL.

\section{Effect of the monomer amount}

TGA was used to determine the grafting percentage of PS onto the DB surface. Before the TGA analysis, the DB/PS nanocomoposite specimens were extracted with the soxhlet extractor. Figure 7 shows the thermogravimetric weight of

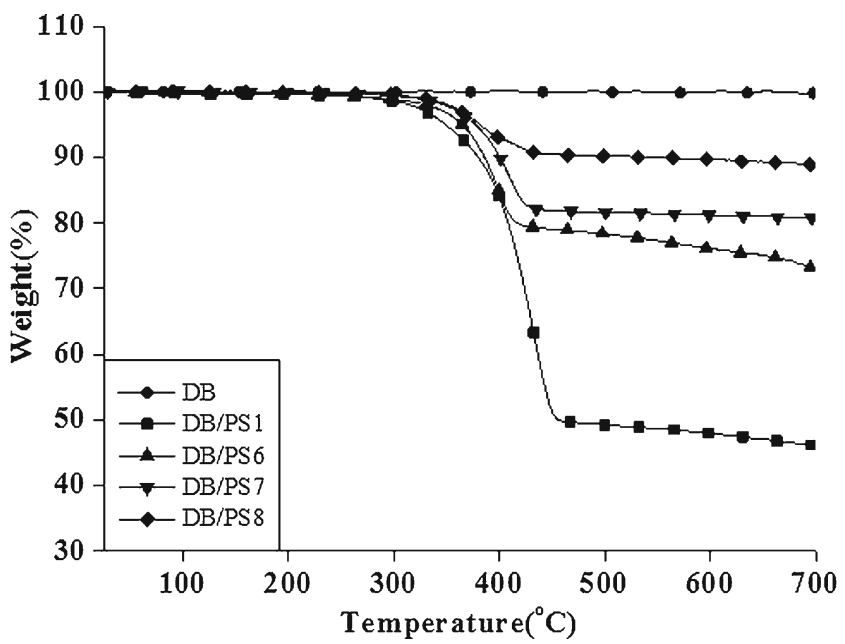

Fig. 8 The TGA curves of DB/PS nanocomposites with different amounts of initiator
DB and the four composites with different amounts of styrene. The obviously weight loss can be found in the temperature range of $300-420{ }^{\circ} \mathrm{C}$ for the $\mathrm{DB} / \mathrm{PS}$ nanocomposites, which were attributed to the decomposition of the polystyrene grafted onto the DB surface. The weight loss of DB/PS nanocomposites decreased with decreasing amount of styrene, which is consistent with the change in electrical resistance.

Effects of the amount of initiator and temperature

Figure 8 plots the TGA curves of the DB/PS nanocomposites with different amounts of initiators. The weight loss of DB/PS1 was the biggest. It was concluded that the optimal amount of initiator was $0.5 \%$ of the monomer, which was consistent with the results of electrical conductivity analysis. Figure 9 shows the weight loss of nanocomposites prepared at different temperatures. As described in table 1, higher polymerizing temperature was favorable to form higher grafting percentage polymer. However, when the temperature was too high, the AIBN would be decomposed.

\section{SEM micrographs}

SEM micrographs are reproduced in Fig. 10 to explain the fracture surface of DB/PS composites which were obtained under different conditions. There was no phase separation for DB/PS1 (Fig. 10a), which indicates the well dispersibility of DB in PS. However, when the amounts of styrene and initiator decreased, clear phase separation could be found on the fracture surface as shown in Fig. 10b, c. Especially as Fig. 10c showed, when the content of initiator was very low, DB aggregates were clearly found.

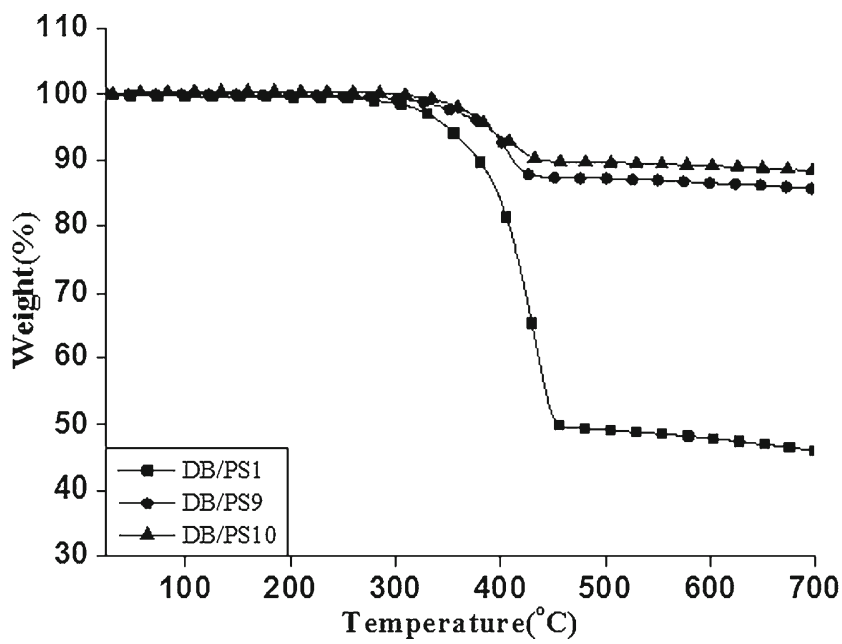

Fig. 9 The TGA curves of DB/PS nanocomposites at different temperatures 

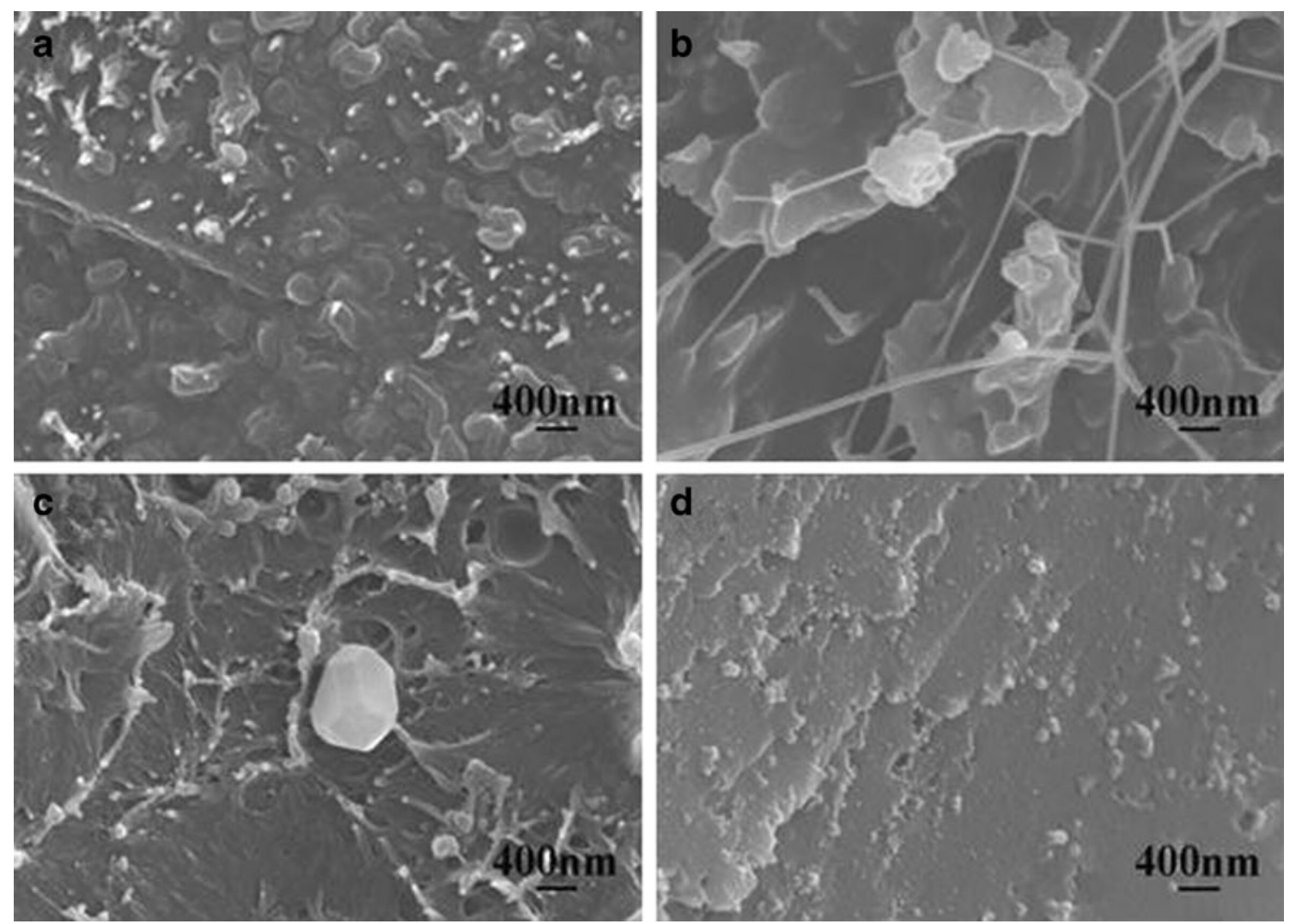

Fig. 10 The SEM images of DB/PS composites obtained at different conditions: (a) DB/PS1, (b) DB/PS2, (c) DB/PS4, and (d) DB/PS6

\section{TEM images}

The PS grafting enabled the DB to be dispersed in the toluene. When pure DB and DB/PS nanocomposites were sonicated in toluene at the same time, the DB started to settle quickly, while the DB/PS nanocomposites remained homogenous with no sedimentation after 2 days.

TEM is also widely used to detect morphology of materials. DB and DB/PS were dispersed in toluene in an ultrasonic bath for $30 \mathrm{~min}$, and then deposited on a copper grid covered with a perforated carbon film. As shown in Fig. 11, pure DB was easily aggregated with aggregates having average diameters of about $30 \mathrm{~nm}$. However, the DB/PS was well dispersed in toluene and the aggregate diameters increased to about $45 \mathrm{~nm}$ after the in situ radical graft polymerization in IL. This indicated that the polystyrene had been successfully grafted onto the DB surface by the proposed method.

\section{Conclusions}

In summary, the preparation of the DB/PS nanocomposites via in situ solution free radical polymerization in IL was
Fig. 11 TEM micrographs of (a) DB and (b) DB/PS1
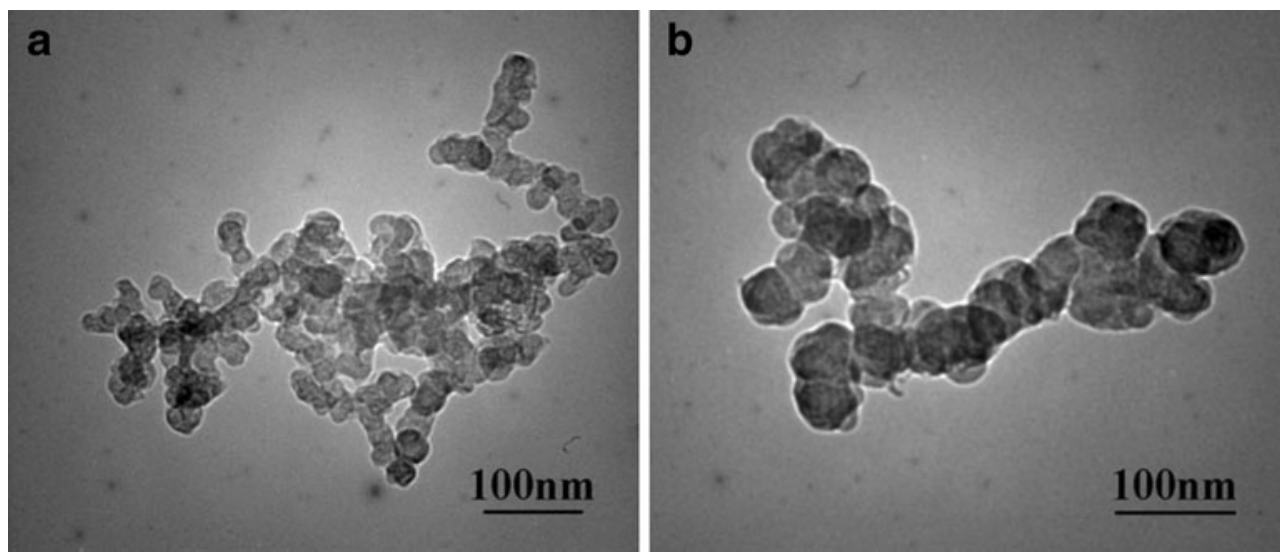
carried out. The nanocomposites separated from the IL because of polystyrene's insobility in the IL. The IL improved the grafting polymerization of styrene on the DB surface. The results of electrical conductivity, FT-IR, SEM and TEM analysis showed that polystyrene grafted and mixed with DB. The electrical conductivity of DB/PS nanocomposites was better than that of pure polystyrene. Both the PG and the conductivity first increased and then decreased with increasing amount of AIBN. And the conductivity decreased and the PG increased with increasing amount of monomer. Furthermore, the conductivity first increased and then decreased with increasing PG. These nanocomposites can be blended with other polymers to get products of improved mechanical properties.

Open Access This article is distributed under the terms of the Creative Commons Attribution License which permits any use, distribution, and reproduction in any medium, provided the original author(s) and the source are credited.

\section{References}

1. Federal Trade Commission (1948) Carbon black export I, et al. Investigations and recommendations under the export trade act. $1245-1345$

2. Peng YK (2007) The effect of carbon black and silica fillers on cure characteristics and mechanical properties of breaker compounds. MS thesis, University Science Malaysia

3. Shen JW, Chen XM, Huang WY (2003) Structure and electrical properties of grafted polypropylene/graphite nanocomposites prepared by solution intercalation. J Appl Polym Sci 88:1864-1869

4. Yasmin A, Luo JJ, Daniel IM (2006) Processing of expanded graphite reinforced polymer nanocomposites. Compos Sci Technol 66:1182-1189

5. Kalaitzidou K, Fukushima H, Drzal LT (2007) A new compounding method for exfoliated graphite-polypropylene nanocomposites with enhanced flexural properties and lower percolation threshold. Compos Sci Technol 67:2045-2051

6. Zheng G, Wu J, Wang W, Pan C (2004) Characterizations of expanded graphite/polymer composites prepared by in situ polymerization. Carbon 42:2839-2847
7. Kim H, Thomas Hahn H, Viculis LM, Gilje S, Kaner RB (2007) Electrical conductivity of graphite/polystyrene composites made from potassium intercalated graphite. Carbon 45:1578-1582

8. Srivastava N, Mehra R (2009) Study of electrical properties of polystyrene/foliated graphite composite. Mater Sci-Poland 27:109-122

9. Azulay D, Eylon M, Eshkenazi O, Toker D, Balberg M, Shimoni N et al (2003) Electrical-Thermal Switching in Carbon-BlackPolymer Composites as a Local Effect. Phys Rev Lett 90:236601

10. Balberg I (2002) A comprehensive picture of the electrical phenomena in carbon black-polymer composites. Carbon 40:139-143

11. Celzard A, McRae E, Mareche J, Furdin G, Dufort M, Deleuze C (1996) Composites based on micron-sized exfoliated graphite particles: electrical conduction, critical exponents and anisotropy. J Phys Chem Solids 57:715-718

12. El Bouazzaoui S, Achour M, Brosseau C (2011) Microwave effective permittivity of carbon black filled polymers: Comparison of mixing law and effective medium equation predictions. J Appl Phys 110:074105-074109

13. Qin F, Brosseau C (2012) A review and analysis of microwave absorption in polymer composites filled with carbonaceous particles. J Appl Phys 111:061301-061324

14. Rohatgi P, Liu Y, Ray S (1992) Friction and wear of metal-matrix composites. Friction and wear of metal-matrix composites. ASM Handbook 18:801-811

15. Radhika N, Subramanian R, Prasat SV (2011) Tribological Behaviour of Aluminium/Alumina/Graphite Hybrid Metal Matrix Composite Using Taguchi's Techniques. Journal of Minerals \& Materials Characterization \& Engineering 10:427-443

16. Zhan Y, Zhang G (2004) Friction and wear behavior of copper matrix composites reinforced with $\mathrm{SiC}$ and graphite particles. Tribol Lett 17:91-98

17. Shim HH, Kwon OK, Youn JR (1990) Friction and wear behavior of graphite fiber reinforced composites. Polym Composite 11:337-341

18. Wasserscheid P, Welton T (eds) (2008) Ionic liquids in synthesis: Wiley Online Library

19. Ma H, Wan X, Chen X, Zhou QF (2003) Reverse atom transfer radical polymerization of methyl methacrylate in imidazolium ionic liquids. Polymer 44:5311-5316

20. Ueda J, Yamaguchi H, Yamauchi T, Tsubokawa N (2007) Radical graft polymerization of vinyl monomers onto nanoparticles in ionic liquid initiated by azo groups introduced onto the surface. J Polym Sci Polym Chem 45:1143-1149

21. Wu XL, Liu P (2010) Polymer grafted multiwalled carbon nanotubes via facile in situ solution radical polymerization. J Exp Nanosci 5:383-389

22. Popov VN, Henrard L, Lambin P (2009) Resonant Raman spectra of graphene with point defects. Carbon 47:2448-2455 